\title{
Long-term consequences of trans-radial catheterization on the radial artery
}

\author{
Nicholas Clarke ${ }^{1}$, Geetha Jagannathan ${ }^{2}$, and Jennifer Lawton ${ }^{2}$ \\ ${ }^{1}$ Johns Hopkins Medicine \\ ${ }^{2}$ Johns Hopkins Hospital
}

September 25, 2021

\begin{abstract}
Background: The radial artery (RA) is often utilized for diagnostic coronary angiography and percutaneous intervention. Recent high-level evidence supports RA use in preference to saphenous vein as a conduit for coronary revascularization. Aim: To demonstrate gross and histologic changes of the RA following transradial access. Methods: We present two patients who had open RA harvest for coronary bypass surgery after transradial catheterization. Results: Examination 8 years after transradial catheterization demonstrated thickened intima and dissection, and examination 12 years following transradial catheterization with percutaneous coronary intervention demonstrated chronic dissection with thickened intima and near occlusion of the lumen. Conclusion: Transradial access via the RA, even after several years, is associated significant injury, making it unusable as a conduit for surgical coronary revascularization. A RA that has been utilized for catheterization should not be considered for coronary revascularization.
\end{abstract}

\section{Long-term consequences of trans-radial catheterization on the radial artery}

Nicholas S. Clarke MD${ }^{1}$, Geetha Jagannathan, $\mathrm{MBBS}^{2}$, Jennifer S. Lawton MD ${ }^{1}$

${ }^{1}$ Division of Cardiac Surgery, Department of Surgery, Johns Hopkins University, Baltimore MD

2 Department of Pathology, Johns Hopkins University, Baltimore MD

Short title: Transcatheter radial artery injury.

Corresponding Author:

Jennifer S. Lawton, MD

1800 Orleans St.

Zayed 7107

Baltimore, MD 21287-0010

Phone: 410-255-2800

Fax: 410-955-3809

Email: jlawton4@jhmi.edu

No source of funding.

IRB Approval: N/A 
Data availability statement: Data sharing not applicable to this article as no datasets were generated or analysed during the current study

\begin{abstract}
Background: The radial artery (RA) is often utilized for diagnostic coronary angiography and percutaneous intervention. Recent high-level evidence supports RA use in preference to saphenous vein as a conduit for coronary revascularization.

Aim: To demonstrate gross and histologic changes of the RA following transradial access.

Methods: We present two patients who had open RA harvest for coronary bypass surgery after transradial catheterization.

Results: Examination 8 years after transradial catheterization demonstrated thickened intima and dissection, and examination 12 years following transradial catheterization with percutaneous coronary intervention demonstrated chronic dissection with thickened intima and near occlusion of the lumen.

Conclusion: Transradial access via the RA, even after several years, is associated significant injury, making it unusable as a conduit for surgical coronary revascularization. A RA that has been utilized for catheterization should not be considered for coronary revascularization.
\end{abstract}

\title{
INTRODUCTION
}

The radial artery (RA) is often used for coronary angiography and percutaneous intervention. With the increased use of total arterial revascularization in accordance with recent guidelines, the RA is more commonly used in coronary artery bypass grafting $(\mathrm{CABG}) .{ }^{1}$ Studies have shown superior long-term patency rates of the RA compared to the saphenous vein. ${ }^{2}$ Questions have been raised as to whether the RA should be used as an arterial graft after transradial (TR) catheterization as it may affect graft patency. Here we discuss two patients with prior TR interventions who after 8-17years, still show structural and histologic changes.

\section{Patient 1}

53-year-old male with a history of kidney ( 8 yrs prior) and liver (17 yrs prior) transplantation and diabetes mellitus complained of chest pain at rest and workup revealed multivessel coronary artery disease (CAD). During left RA dissection, the distal portion was thickened, and a chronic dissection flap was noted (Figure 1) .

\section{Patient 2}

48-year-old female with a history of CAD (PCI and stents 12 yrs prior), diabetes mellitus, hyperlipidemia, and statin intolerance presented with dyspnea and chest pain. Nuclear stress test was positive and cardiac catheterization demonstrated multivessel CAD. The entire the RA was noted to have a chronic dissection (Figure 2 ). And it was sent to pathology for histologic examination. Findings were significant for a portion of the lumen occluded by fibrosis and recanalization (Figure 3 ).

\section{DISCUSSION}

Here we present two patients with more than 8 years after TR arterial line or catheterization with chronic injury in the form of dissection and obstruction of the lumen due to fibrosis. Current evidence supports that TR procedures cause chronic and irreparable injury to the RA, making them unusable as bypass conduit for CABG.

Sousa-Uva and colleagues recommend that the RA should de facto be considered in every CABG. ${ }^{3}$ The group cites the superior late graft patency compared with traditionally harvested saphenous vein, as well as, stronger evidence of clinical benefit when compared to the right internal thoracic artery.

Gaudino summarized the benefits of using the TR approach for percutaneous procedures and the RA as a conduit for CABG. ${ }^{4}$ The writing panel comprised of clinical cardiologists, cardiothoracic surgeons, and 
interventional cardiologists recommended reserving one RA for TR access and the other as a conduit for CABG. They also recommended adoption of strategies to minimize RA damage during TR access.

To date there are only two studies that specifically examine the impact of previous TR procedures on the function of RA used as conduit in CABG. Kamiya reported a stenosis- free patency rate of $77 \%$ in those with prior RA catheterization versus $98 \%$ in the control group (no prior RA catheterizations) at 30 days. ${ }^{5}$ The authors also performed a subanalysis on the relationship between occurrence of graft stenosis and TR catheterization, which indicated that the number of previous TR catheterizations was the most likely factor affecting graft patency $(\mathrm{P}=0.07)$. In a similar study, Ruzieh reported a 6 - to 18 -month patency of $59 \%$ in the TR access group compared to $78 \%$ in the control group (RA not used for angiography) ( $\mathrm{p}=0.03){ }^{6}$

\section{TR access is associated with endothelial injury and intimal hyperplasia.}

Kamiya noted intimal hyperplasia (the primary method of long-term saphenous vein graft failure) in $68 \%$ of RAs in the prior RA catheterization group and $39 \%$ in those from the control group $(\mathrm{P}=0.046)$. Gaudino ${ }^{7}$

examined 50 patients who underwent TR coronary angiography before CABG and split the patients into 3 groups depending on the time interval. Immunohistochemical analysis demonstrated extensive endothelial injury in all examined RAs, with a trend toward a reduction in damage over time.

Nitroglycerin-mediated dilation (NMD) and flow-mediated dilation (FMD) are frequently used to study vasomotor function of the RA. Burstein found that, although the NMD response showed some trend for recovery over time, the FMD response was almost completely abolished after 9 weeks. ${ }^{8}$ Yan demonstrated that TR procedures decreased RA NMD and FMD resulting in immediate and persistent blunting of vasodilatory function. ${ }^{9}$

Using ultrasound imaging, studies have shown that the diameter of the RA following TR intervention never completely returns to baseline. ${ }^{10} \mathrm{~A}$ meta-analysis by Rashid found that, following TR intervention, the incidence of RA occlusion within 24 hours was $7.7 \%$, which decreased to $5.5 \%$ at $>1$ week follow-up. ${ }^{11}$. The only intervention that significantly reduced the risk of occlusion was use of a higher dose of heparin $(5,000$ IU vs $<5,000)$.

\section{Conclusion}

Studies evaluating the durability of the RA as a conduit after TR intervention are very limited, and few have demonstrated that injury remains after many years. We demonstrate that after multiple years following TR intervention, the RA shows persistent injury and should not be utilized as a conduit for CABG. Unfortunately, chronic injury to the RA prevents future use as a surgical revascularization conduit, thereby depriving the patient of its well documented benefits of improved patency and prolonged survival.

\section{References}

1. Neumann FJ, Sousa-Uva M, Ahlsson A, et al. 2018 ESC/EACTS Guidelines on myocardial revascularization. European heart journal. Jan 7 2019;40(2):87-165.

2. Gaudino M, Benedetto U, Fremes S, et al. Radial-Artery or Saphenous-Vein Grafts in Coronary-Artery Bypass Surgery. The New England journal of medicine. May 31 2018;378(22):2069-2077.

3. Sousa-Uva M, Gaudino M, Schwann T, et al. Radial artery as a conduit for coronary artery bypass grafting: a state-of-the-art primer.European journal of cardio-thoracic surgery : official journal of the European Association for Cardio-thoracic Surgery . Dec 1 2018;54(6):971-976.

4. Gaudino M, Burzotta F, Bakaeen F, et al. The Radial Artery for Percutaneous Coronary Procedures or Surgery? Journal of the American College of Cardiology . Mar 13 2018;71(10):1167-1175.

5. Kamiya H, Ushijima T, Kanamori T, et al. Use of the radial artery graft after transradial catheterization: is it suitable as a bypass conduit? The Annals of thoracic surgery . Nov 2003;76(5):1505-9. 
6. Ruzieh M, Moza A, Siddegowda Bangalore B, Schwann T, Tinkel JL. Effect of Transradial Catheterisation on Patency Rates of Radial Arteries Used as a Conduit for Coronary Bypass. Heart Lung Circ . Mar 2017;26(3):296-300.

7. Gaudino M, Leone A, Lupascu A, et al. Morphological and functional consequences of transradial coronary angiography on the radial artery: implications for its use as a bypass conduit. European journal of cardio-thoracic surgery : official journal of the European Association for Cardio-thoracic Surgery . Sep 2015;48(3):370-4.

8. Burstein JM, Gidrewicz D, Hutchison SJ, Holmes K, Jolly S, Cantor WJ. Impact of radial artery cannulation for coronary angiography and angioplasty on radial artery function. The American journal of cardiology . Feb 15 2007;99(4):457-9.

9. Yan Z, Zhou Y, Zhao Y, Zhou Z, Yang S, Wang Z. Impact of transradial coronary procedures on radial artery function. Angiology. Feb 2014;65(2):104-7.

10. Mounsey CA, Mawhinney JA, Werner RS, Taggart DP. Does Previous Transradial Catheterization Preclude Use of the Radial Artery as a Conduit in Coronary Artery Bypass Surgery? Circulation . Aug 30 2016;134(9):681-8.

11. Rashid M, Kwok CS, Pancholy S, et al. Radial Artery Occlusion After Transradial Interventions: A Systematic Review and Meta-Analysis.J Am Heart Assoc. Jan 25 2016;5(1)doi:10.1161/jaha.115.002686

\section{Hosted file}

Figure 1.eps available at https://authorea.com/users/437432/articles/539073-1ong-termconsequences-of-trans-radial-catheterization-on-the-radial-artery 
figures/Figure-2/Figure-2-eps-converted-to.pdf 
figures/Figure-3/Figure-3-eps-converted-to.pdf 